\title{
Verbos de movimiento en un diccionario bilingüe sintáctico-combinatorio francés-español: TACTICOMB
}

\section{Motion verbs in a bilingual syntactic-combinatorial dictionary in French and Spanish: TACTICOMB}

\author{
Gemma Sanz EsPinar \\ Universidad Autónoma de Madrid \\ gema.sanz@uam.es
}

\begin{abstract}
After Talmy (1985) and Slobin (2004) highlighted the existence of tendencies in conceptualization and lexicalization of motion events in languages, there has been a lot of research on this topic in different areas: Linguistics, Translation Studies, First and Second Language Acquisition or Second Language Teaching. As Talmy points out, the expression of motion has different tendencies in languages from highlighting the trajectory of motion to highlighting the manner of motion in the verb, but this is not the only factor (linguistic) that allows to make some notions explicit or to keep others implicit, in a discursively acceptable utterance (Slobin). Our aim is to try to transfer this kind of lexical knowledge into a bilingual syntactic-combinatorial dictionary, TACTICOMB, through the class of motion verbs that do not seem to involve, in their "literal" senses, a restricted combinatory (collocations) as appears in many combinatory dictionaries.
\end{abstract}

\begin{abstract}
Résumé
Après les études de Talmy (1985) et de Slobin (2004) montrant les tendances dans la conceptualisation et la lexicalisation des procès de mouvement dans les langues, il y a eu de nombreuses recherches à ce sujet provenant de la Linguistique, la Traduction, l'Acquisition de la langue maternelle et étrangère ou la Didactique des langues étrangères. Comme le signale Talmy, l'expression du mouvement se fait différemment selon les langues, elles expriment dans le verbe soit la trajectoire, soit la manière du mouvement. Or ce n'est pas le seul facteur qui détermine la confluence d'información sur le mouvement dans un énoncé totalement acceptable dans la langue (Slobin). Notre objectif est de transposer ce type de connaissance lexicale à un dictionnaire syntactico-combinatoire bilingue, TACTICOMB. On l'illustrera pour la classe de verbes de mouvement qui ne semblent pas impliquer de combinatoire restreinte, dans leurs acceptions "littérales", comme c'est le cas pour beaucoup des dictionnaires de combinatoire.
\end{abstract}


Anales de Filología Francesa, n. ${ }^{\circ} 28,2020$

VERBOS DE MOVIMIENTO EN UN DICCIONARIO BILINGÜE SINTÁCTICO-COMBINATORIO...

\section{Keywords}

motion event, conceptualization, lexicalization, combinatory, bilingual syntactic-combinatorial dictionary.

\section{Mots-clés}

procès de mouvement, conceptualisation, lexicalisation, combinatoire, dictionnaire syntactico-combinatoire bilingue.

\section{Introducción}

El estudio de los fenómenos de coocurrencia o coaparición de palabras en el discurso, de la combinatoria en sentido amplio o bien restringido (colocaciones), ha adquirido en la última treintena de años una dimensión central no solo en Lingüística, sino también en Traducción o Didáctica de las lenguas extranjeras.

Por otro lado, el estudio de las distintas características de los verbos de movimiento en diferentes lenguas se ha estudiado ampliamente en Lingüística contrastiva y tipológica (Talmy, 1985; Morimoto, 2001; Pourcel \& Kopecka, 2005; Kopecka, 2006), pero también en otros ámbitos donde el contacto o contraste entre lenguas es prácticamente inherente, como la Traducción (Slobin, 2004; Cifuentes Férez \& Rojo, 2015), la Adquisición de Lenguas Maternas y Extranjeras (Berman \& Slobin, 1994; Hickmann et al., 1998; Noyau et al., 2005; Sanz Espinar, 2004; Sanz et al., 2012) o la Didáctica de Lenguas Extranjeras (Bylund \& Athanasopoulos, 2015).

En este artículo adoptaremos un enfoque cognitivo, textual-discursivo ${ }^{1}$ y comparado de la combinatoria relacionada con la expresión del movimiento (a través de los verbos de movimiento en francés y español) con el fin de contribuir a la reflexión sobre el diseño de un diccionario bilingüe sintáctico-combinatorio que sirva para la producción y la traducción tanto como para la comprensión y aprendizaje y que llamamos TACTICOMB (Sanz \& Gil, 2019). Este diccionario se encuentra aún en fase exploratoria en su diseño, que será además digital $^{2}$.

Los diccionarios combinatorios monolingües tienen ya un recorrido importante desde los años 80 y tenemos diseños variados: REDES o DICE ${ }^{3}$ en español o DEC en francés, con un carácter más especializado, vs. PRACTICO en español y LR o GDdesCOOC en francés, para gran público. Barrios (2008) llama a REDES “diccionario de restricciones léxicas”, por oposición a PRÁCTICO, “diccionario de producción” para gran público (que, dice, ayuda a encontrar la palabra adecuada que acompañe a otra) y advierte de que la frecuencia no es el criterio que sigue ninguno de ellos, pero sí es un criterio, por ejemplo, para un diccionario de

1 Haremos la distinción textual vs. discursivo siguiendo a Adam (1997) entre el nivel textual (donde se construye la coherencia y la cohesión, sin las condiciones de producción) y el discursivo (el discurso se entiende como el texto con sus condiciones de producción). Aunque hablaremos de género discursivo (como sinónimo de género textual), por lo corriente del término, para remitirnos a los tipos de texto con una estructura de contenido y forma convencional y reconocible.

2 Nuestro objetivo es un diccionario en línea que será publicado en el futuro en https://www.dicos.es.

3 Remitimos a todos los diccionarios mediante unas siglas o nombre abreviado. Al final de nuestra bibliografía se encuentra las referencias completas a las que se accede a través de los nombres abreviados. 
lengua general como el de Oxford, que recoge good book o great book, por ser combinatoria muy frecuente de la palabra book.

Ahora bien, hasta donde conocemos, no disponemos todavía de diccionarios o proyectos de diccionarios combinatorios bilingües que tengan el español y el francés como una de las dos lenguas L1 o L2 ${ }^{4}$ presentes, salvo por los siguientes casos: el diccionario combinatorio EDCEI español-italiano de Greco (2015), la descripción del diseño de Lépinette (1989) de la adaptación del DEC (Mel'čuk et al., 1984-1999) para construir un diccionario francésespañol basado en la teoría de texto-sentido de Mel'čuk (Mel'čuk \& Milićević, 2020) y la base de datos de colocaciones francés-español que diseña Alonso (2001). Creemos que esto puede estar motivado por:

1) la complejidad del fenómeno de la combinatoria, que exige, además, una metodología laboriosa para su identificación (en general, a día de hoy, a menudo basada en corpus). Comprobamos que, según se defina el propio término de combinatoria, los verbos de movimiento se pueden ver excluidos de los diccionarios combinatorios. Así, de los mencionados anteriormente, sólo PRÁCTICO recoge estos verbos ${ }^{5}$,

2) los niveles afectados por la combinatoria y la dificultad que plantea articular y presentar información de distinta naturaleza (sintáctica, semántica y combinatoria léxica) de dos lenguas, de un modo que pueda procesar un usuario no especialista en lingüística.

La visualización de la información en el diccionario resulta compleja porque hay otros elementos en juego si tenemos en cuenta un objetivo de producción o traducción:

a) factores de tipo textual-discursivo, que afectan a la combinatoria: la formulación del enunciado y su cotexto ${ }^{6}$, los elementos de cohesión y coherencia, las características del propio género discursivo oral o escrito,

b) atender a la especificidad de las operaciones de comprensión, producción, traducción o aprendizaje, es decir, la utilidad del diccionario.

Es cierto, como dice Alonso (2001), que los diccionarios de combinatoria suelen concebirse como diccionarios de ayuda a la producción en la lengua que describen; ahora bien, en nuestro caso, el objetivo de la traducción es primordial por tratarse de un diccionario bilingüe. Por ello, vamos a tratar de reflexionar y ahondar en una estructura intermedia

4 A lo largo del artículo usaremos L1 como la lengua de las entradas en cada una de las dos partes del diccionario bilingüe. Es decir, tanto si es español $>$ francés como si es francés $>$ español, la lengua que aparezca ordenada alfabéticamente en las entradas será la L1. La L2 será la lengua de destino a la que se llega a través de la L1.

5 Algunas ausencias notables en PRÁCTICO en lo que concierne a los verbos de movimiento que hemos observado son los verbos bajarse, desplazar, desplazarse, escaparse, marcharse, penetrar, subirse, trepar, volver. Sí figuran, en cambio: abandonar, acercar, acercarse, adentrarse, alejar, alejarse, arrojar, arrojarse, ascender, avanzar, bajar, caer, caerse, correr, cruzar, despeñar, despeñarse, entrar, escapar, huir, hundir, hundirse, introducirse, ir, irse, lanzar y lanzarse, llegar, marchar, pasar, precipitarse, retroceder, saltar, salir, subir, tirar, tirarse, venir, zarpar.

6 Entenderemos por cotexto, el entorno lingüístico más o menos cercano que determina el sentido de una palabra, frase o fragmento. No solo dentro de una misma frase, sino en frases contiguas. 
cognitivo-conceptual entre las lenguas que ponga de manifiesto la no simetría de las lenguas. Esto nos parece importante porque la propuesta que hemos encontrado en el diccionario bilingüe español- italiano EDCEI asocia una lexía a otra y se da una muy falsa apariencia de "reversibilidad" que nos gustaría evitar.

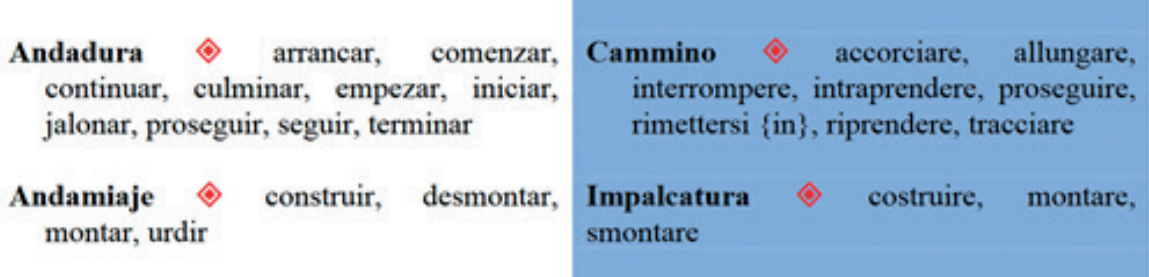

Figura 1. Extracto del diccionario EDCEI (Greco, 2015: 211).

Trataremos de abordar esa "interfaz cognitiva" que queremos construir, a través de lo que llamaremos una mesoestructura en el diccionario, de manera que se facilite la identificación de esquemas conceptuales propios de cada lengua, no simétricos, pero sí que permitan el paso de una lengua a la otra. En este sentido, queremos indicar que el diccionario bilingüe, por su naturaleza, lleva a menudo al lector poco avezado a dar por supuesta una simetría (Sanz Espinar, 2004). Es evidente que, si la utilidad del diccionario es la de ayudar a la traducción, se trata de encontrar equivalencias totales y válidas, pero también es verdad que precisamente las lenguas no son simétricas. En este sentido, nos gustaría en la medida de lo posible aumentar tanto las posibilidades de encontrar opciones válidas con la mayor equivalencia de sentido posible, como aumentar las opciones válidas con "menor grado de equivalencia semántica”, pero "mayor grado de equivalencia pragmática”.

Con todo, no queremos desatender la comprensión y el aprendizaje, y en especial el aprendizaje lo entenderemos sobre todo como "mejora de la comprensión" del contraste L1L2. Algunos autores como Bylund \& Athanasopoulos (2015), se han planteado la aprendibilidad y, por ende, la enseñabilidad del tipo de patrones combinatorios que vamos a presentar aquí. En concreto, para estos autores, solo podrán aprenderse los contrastes en la conceptualización (thought) y la expresión del movimiento entre lenguas si el profesor es consciente de estos contrastes y sabe elegir materiales y tareas, entre otras de (re)conceptualización, para hacer explícitos dichos contrastes. En caso contrario, el aprendiz puede tardar demasiado en captar estos matices. También abogan por el uso de imágenes en movimiento (escenas filmadas de movimiento) para favorecer la adquisición de nuevos esquemas de conceptualización mediante una metodología multimodal y nos recuerdan que estamos en una época de reconocimiento de la relatividad lingüística y de la pedagogía plurilingüe y pluricultural. Así, para ellos, el reto es el siguiente: 
An important task for policy makers, curriculum designers, and practitioners is consequently to decide what to do with this knowledge. At least two questions should be discussed in this regard: Is it desirable to include the acquisition of language-specific thought patterns in the curricular goals? Can the knowledge about language specificity in thought be used in teaching as a means to facilitate learning? (Bylund \& Athanasopoulos, 2015: 8)

En nuestro caso, respondemos afirmativamente a la primera pregunta. Enseñar estos patrones forma parte de los objetivos deseables de aprendizaje de una lengua extranjera, aunque la edad, progresión y objetivos progresivos estarían por determinar y, por otro lado, proponemos la explicitación y ordenación de este conocimiento, en un formato de diccionario combinatorio, con el que identificar, comprender y aprender la relatividad lingüística en lo que se refiere a los verbos de movimiento.

A lo largo del artículo, realizaremos una recopilación de distintos factores que inciden en la expresión de un evento de movimiento, algunos de orden lingüístico y semánticocognitivo (a nivel del enunciado) y otros de tipo textual y discursivo (coherencia y cohesión identificable en la producción real). Con ello plantearemos cómo podríamos ordenar la información en un diccionario sintáctico-combinatorio bilingüe que actúe como un léxicogramática cognitivo.

\section{La clase de verbos de movimiento: estudios cognitivos, tipológicos y contrastivos}

Vamos a comenzar por describir el enfoque del que partimos y por reunir datos lingüísticos contrastados sobre la expresión del movimiento en español y francés.

En primer lugar, en el nivel semántico-cognitivo, nos apoyaremos en algunos conceptos básicos del modelo de producción oral de Levelt (1989). Para Levelt, hablar implica una serie de operaciones mentales que se desencadenan a partir de una intención de comunicación que surge en el hablante. Desde la intención, durante la producción oral, se ha de ir construyendo una coherencia mediante distintas operaciones en el nivel de la conceptualización: la selección de la información para ser explicitada, su secuenciación y su jerarquización, todo lo cual resulta en un mensaje preverbal. Este mensaje tiene dos características:

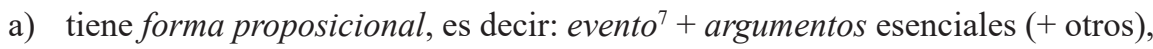

b) tiene una relación directa con el léxico, ya que en la conceptualización (selección de información para ser explicitada) juega un papel preponderante la selección léxica, en especial, la del verbo que expresa el evento.

7 Evento, en el sentido francés de procès: situación conceptualizada para un periodo de tiempo, que tiene una estructura argumental y es expresada normalmente por verbos. En la conceptualización, la selección del léxico verbal refleja el filtro conceptual de la realidad, pues se remite a microsituaciones que reflejan la selección de informaciones para ser explicitadas frente a lo que no se explicita. 
Una vez seleccionado el contenido léxico, se opera la formulación (aplicación de una sintaxis y de una morfología al léxico, en especial, en el caso de palabras variables, i.e. se trata de la selección de las formas flexivas adecuadas y de la ordenación lineal en la proposición). Por último, tiene lugar la articulación propiamente dicha (tratamiento por el aparato fonador para la emisión efectiva del mensaje).

En el esquema que vemos en el ejemplo (1) podemos identificar los dos niveles esenciales de dicho modelo. Ambos se encuentran determinados por la lengua utilizada: la selección de información para ser explicitada (conceptualización) y la selección de los elementos léxicos y morfosintácticos (lexicalización/formulación).

El reto para cualquier diccionario bilingüe es precisamente poder reflejar y explicitar que hay una conceptualización potencial o necesariamente "diferente" aunque pueda encontrarse una correspondencia o equivalencia semánticamente cercana en la L2.

En el ejemplo (1), para el español y el francés se muestra una estructura similar, pero vemos que a la hora de expresar la meta del desplazamiento en francés se da una especie de redundancia "obligatoria", pues tanto en el verbo como en el complemento localizador se marca de manera duplicada ese rasgo que hemos llamado supra, mientras que en español precisamente "sobraría" por redundante (solo se sube "hacia arriba").

$\begin{array}{llll}\begin{array}{l}\text { FIGURA } \\ \text { (que se desplaza) }\end{array} & \text { TRAY (dirección) } & \begin{array}{l}\text { META (+supra) } \\ \text { (del desplazamiento) }\end{array} & \text { FINALIDAD } \\ \text { | } & \mid & \text { C. Lugar } & \text { Subordinada } \\ \text { Sujeto } & \mathrm{V} & \text { al árbol } & \text { para ver los polluelos. } \\ \text { El niño } & \text { subió } & \text { sur l'arbre } & \text { pour voir les oisillons. } \\ \text { L'enfant } & \text { est monté } & & \end{array}$

Talmy (1985), desde un enfoque lingüístico tipológico, identifica esquemas o patrones de conceptualización y de lexicalización específicos en distintos campos (o ámbitos) referenciales, como el movimiento. La lexicalización (en este enfoque) se puede considerar como la totalidad del material lingüístico (léxico o eventualmente morfológico), es decir, viene a corresponder con la forma (o formulación) del mensaje (unidades léxicas, prefijos, partículas, preposiciones).

Un patrón de conceptualización sería una coaparición o, en términos de Talmy, una confluencia de rasgos semánticos con cierto grado de sistematicidad en las proposiciones, y en algunas unidades léxico-gramaticales en particular, a la hora de hacer referencia a un dominio de referencia, como la dinámica de la fuerzo o del movimiento. Así, por ejemplo, es posible que en una lengua en la proposición que introduce un cambio de lugar en el discurso esto confluya con la conceptualización de un evento de LOCALIZACIÓN: Y luego está en el jardín... 
LOCALIZACIÓN télica: Y luego aparece en el jardín... o de MOVIMIENTO no télico ${ }^{8}: Y$ luego va al jardín o de MOV+DIRECCIÓN: Y luego sale al jardín o de AGENTIVIDAD: $Y$ luego le hace salir al jardín. Por otro lado, se encuentran los patrones de lexicalización: tipos de unidades léxicas o gramaticales que se combinan de manera sistemática, recurrente para expresar un mismo patrón conceptual. Por ejemplo, en español, es prototípico para MOVtéli co+IN+AGENTIVIDAD+ENTIDAD ANIMADA el uso del verbo simple meter (meter a alguien en un furgón), mientras que en francés podemos encontrar verbos prefijados o perífrasis con faire (faire rentrer, emmener, faire monter... quelqu'un dans un fourgon).

Para las situaciones de movimiento, Talmy identificó inicialmente dos tipos de lenguas: unas que marcan el movimiento (cuyo rasgo semántico básico es la TRAYECTORIA) en el verbo y otras, que lo marcan en una palabra (partícula, preposición o adverbio) que él llama satélite. De ahí, la oposición, inicialmente solo binaria, entre las lenguas que él llama lenguas de marco verbal (lenguas románicas) y lenguas de marco satélite (inglés). Desde el famoso ejemplo de Talmy, The bottle floated out of the cave, cuyo equivalente en español sería La botella salió de la cueva flotando y en francés La bouteille est sortie de la grotte en flottant, ha habido algunas evoluciones importantes en esta clasificación de lo que serían asociaciones entre los patrones de conceptualización y de lexicalización (funciones sintácticas que se les asocian).

En nuestro diccionario creemos útil contar con una mesoestructura de tipo cognitivo, que pueda reunir características globales de la clase semántica de los verbos de movimiento de cada lengua (es decir, un contraste generalizable a nivel conceptual), de modo que esto pueda explicar, al menos parcialmente, el nivel de la formulación o lexicalización. Para esto podemos contar con un listado virtual de los elementos o rasgos semántico-conceptuales que pueden potencialmente entrar en juego en la expresión de un movimiento. Por "virtual" entendemos que en el discurso aparecerán una selección de ellos, potencialmente diferente según la lengua:

FIGURA (figure) la entidad que se mueve con respecto a otro FONDO (ground) otro objeto respecto al cual se mueve la figura

TRAYECTORIA O DIRECCIÓN (path) del movimiento ORIGEN (origin) del desplazamiento

META (goal) lugar al que se llega tras el desplazamiento MANERA (manner) del movimiento

En el ejemplo (2) se muestra una frase que expresa el movimiento en inglés. Añadimos debajo el análisis del esquema de conceptualización correspondiente, lo que funciona

8 El concepto de télico implica marcar una frontera o cambio de estado, es decir, en el caso del movimiento se trataría de una terminación/conclusión, más que un mero cese, del movimiento (un cese no implicaría telicidad implícita en el verbo). Por ejemplo, en español, correr no es nunca cualitativamente télico, necesita de elementos externos cuantitativos para marcar una frontera temporal: dejar de correr). 
precisamente como una mesoestructura (confluencia de varios semas en la proposición y distribución en distintas unidades léxicas con funciones sintácticas diferentes). Tomando como ejemplo principal el inglés, se pone de manifiesto el contraste entre una lengua de marco verbal (francés y español) y una lengua de marco satélite (inglés). En este caso, el español y el francés aparecen como "lenguas de traducción" y comentaremos las particularidades de la conceptualización en cada opción de traducción. En el diccionario, los ejemplos en francés y español aparecerían también con sus glosas para cada lengua, como en el ejemplo (3), de modo que se enriquezca la mesoestructura.

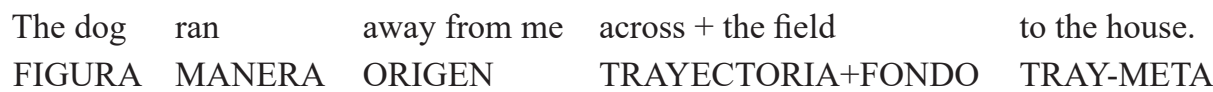

Traducciones "cercanas":

ES1. El perro se alejó de mí ES1a) corriendo ??a través del campo hacia la casa.

ES1b) cruzando/atravesando el campo a la carrera hacia la casa.

ES1c) corriendo campo a través hacia la casa

ES2. El perro se alejó de mí y cruzó el campo corriendo en dirección a la casa/??hacia la casa. ES3. El perro se alejó de mí y corrió campo a través en dirección a la casa/??hacia la casa. FR1. Le chien s'est éloigné de moi en courant à travers le champ vers la maison.

FR2. Le chien s'est éloigné de moi et a traversé le champ en courant vers la maison.

FR3. Le chien s'est éloigné de moi et a couru à travers ( ?le) champ vers la maison.

En el ejemplo (2) vemos cómo en inglés confluyen en una proposición "simple" (un solo evento al que corresponde un solo verbo) TODOS los elementos mencionados para el movimiento (figura, manera, origen, trayectoria, fondo y meta). En cuanto a la confluencia de semas destaca que el verbo expresa la MANERA y los complementos de lugar, el ORIGEN, la TRAYECTORIA+FONDO y la META, cada uno con preposición o combinación de preposiciones diferentes.

Nuestras propuestas de traducción "cercanas" a español y a francés remiten a varias opciones que tratan "a toda costa" de no perder información semántica a la hora de trasladarla a otra lengua.

En español y francés hemos optado por no poner la manera en el verbo. Correr es una actividad o manera de moverse, mientras que alejarse de es un verbo que permite marcar la dirección topológica respecto de un punto de origen que es YO $(m i)$. Después, hemos jugado con varias opciones para tratar de conservar, por un lado, la manera, por otro, la trayectoria $\mathrm{y}$, por último, la meta, a costa de reformular, es decir, de cambiar las categorías léxicas que expresan la trayectoria (de la preposición accross al verbo cruzar o atravesar o a la locución 
adverbial a través: accross the field > cruzando/atravesando el campo // campo a través). En todas las opciones ES1a, ES1b, ES1c hemos utilizado el gerundio que Talmy indica como tendencia tipológica de las lenguas románicas.

Sin embargo, en ES1a nos topamos con algo que "no suena bien" (Se alejó de mí corriendo ? a través del campo hacia la casa) salvo que hagamos pausas importantes (Se alejó de mí // corriendo a través del campo hacia la casa). Es como si corriendo no pudiera a la vez indicar la manera de alejarse y ser verbo de una proposición subordinada con sus propios complementos (a través del campo hacia la casa). Es decir, la entonación marca una pérdida de autonomía de corriendo cuando se combina con un verbo de dirección, lo que indica una lexicalización del conjunto VDIR+VMAN. La opción ES1b (cruzando el campo a la carrera hacia la casa) adjunta la manera del movimiento (a la carrera) al verbo cruzar y no a alejarse de mí. Se trata de un cambio algo arriesgado en una traducción porque modifica ya la distribución de semas en las unidades léxicas de modo importante, aunque gracias a que usamos a la carrera en vez de corriendo no se produce el efecto de la frase ES1, evitando abusar de gerundios (?cruzando el campo corriendo). En ES2 y ES3 proponemos una división en dos proposiciones independientes y principales, una que marca el origen y la telicidad y otra que contiene lo demás, lo que aligera en parte la secuencia. Ahora bien, si cambiamos el uso de hacia la casa por en dirección a la casa observamos que la frase mejora todavía más. Lo mismo ocurre si utilizamos corriendo campo a través en vez de corriendo a través del campo. Es aquella una opción propia del español, inexistente en francés, que se puede activar en una traducción del inglés al español si fuera necesario: cayó escaleras abajo, subió escaleras arriba, se precipitó colina abajo/arriba, campo a través... Permite precisamente la confluencia del fondo y de la trayectoria con subespecificaciones topológicas9.

Podemos también observar que la frase en inglés no marca la "llegada a la meta". En inglés tendríamos más bien ran up to the house, para que el evento fuese "télico", en cuyo caso se marcaría "otra frontera", dado que al decir ran away from me ya se ha introducido una "frontera" que hace el evento télico (away from me). En español y francés, la frase subordinada parece "mejorar" si ponemos hasta la casa, de modo que se marque la meta del movimiento. Como vemos, al tener que recurrir a dos verbos en español y francés, hemos de plantearnos si ambos van a marcar o no una "telicidad", dada la frase de partida que solo tiene una. Una vez más, dependiendo del cotexto inmediatamente posterior, podremos traducir marcando dos fronteras si esa segunda frontera estuviera implicada o explicitada en alguna frase posterior (reformulación suprafrástica), y esto es posible porque un "evento de movimiento" está inscrito en el tiempo y la expresión de dicho movimiento (por medio, no solo, de verbos, sino del contenido proposicional en su conjunto) conllevará características temporales precisas.

9 La Nueva gramática de la lengua española de la RAE indica combinaciones entre otros de sustantivos como campo/camino/calle/pendiente/escaleras/rio/pasillo/pierna y los adverbios de trayectoria abajo/arriba/a través/adelante/atrás. Se precisa así "hacia dónde" (subespecificaciones topológicas). 
En francés, hemos hecho una serie de propuestas de traducción similares a las del español. En FR1, Le chien s'est éloigné de moi en courant à travers le champs vers la maison, se marca la manera de $s$ 'éloigner por medio del gerundivo (en+participio presente) y la frase describe el desplazamiento. En FR2 el sentido varía un poco, porque se adjunta la manera de moverse (en courant) a traverser, en vez de a s'éloigner, el foco pasa por tanto a la meta y en cómo se dirige el perro hacia la meta. En FR3, por la falta de gerundio, el verbo se presta a utilizar la expresión a couru à travers champ, pero cualitativamente esta opción indica precipitación y “tomar el camino más rápido/un atajo” (podría equivaler según los contextos a se precipitó directo hacia la casa).

En definitiva, tras este análisis, podemos presentar una serie de frases contrastadas que permitirían visualizar el contraste de patrones de conceptualización en el diccionario (lo que formaría parte de la mesoestructura).

\section{3.a INGLÉS}

The dog ran

away from me

across + the field

to the house

FIGURA MANERA

TRAY+ORIGEN

TRAYECTORIA+FONDO

TRAY + META

\section{3.b ESPAÑOL}

3.b.1. El perro se alejó de mí corriendo campo a través hacia la casa

FIGURA TRAY ORIGEN MANERA

TRAY+FONDO TRAY +META

\begin{tabular}{|c|c|c|c|}
\hline 3.b.2. & El perro & se alejó & de mí \\
\hline & FIGURA & TRAY & ORIGEN \\
\hline & y cruzó el campo & corriendo & en dirección a la casa \\
\hline & TRAY+FONDO & MANERA & TRAY +META \\
\hline
\end{tabular}

\section{Diferencias:}

Corte de la frase: cambio de ritmo y estilo, pero más fluido que 3.b.1.

La manera (corriendo) se ha aplicado a otro verbo (cruzar).

\begin{tabular}{|c|c|c|}
\hline El perro & se alejó & de mí \\
\hline FIGURA & TRAY & ORIGEN \\
\hline y corrió & campo a través & en dirección a la casa. \\
\hline MANERA & TRAY+FONDO & TRAY+META \\
\hline
\end{tabular}

\section{Diferencias:}

Corte de la frase: cambio de ritmo y estilo. 
La expresión campo a través tiene a menudo un matiz literario o está geográfica mente determinada en castellano.

\section{3.c FRANCÉS}

3.c.1. Le chien s'est éloigné de moi en courant à travers le champ vers la maison. FIGURA TRAY ORIGEN MANERA TRAY+FONDO TRAY+META

3.c.2. Le chien s'est éloigné de moi

FIGURA TRAY ORIGEN

et a traversé le champ en courant vers la maison.

TRAY+FONDO MANERA TRAY+META

\section{Diferencias:}

Corte de la frase: cambio de ritmo y estilo, pero más fluido que 3.c.1.

La manera (corriendo) se ha aplicado a otro verbo (cruzar).

$\begin{array}{llll}\text { 3.c.3. } & \text { Le chien } & \text { s'est éloigné } & \text { de moi } \\ \text { FIGURA } & \text { TRAY } & \text { ORIGEN } \\ \text { et a couru } & \text { à travers champ } & \text { vers la maison. } \\ \text { MANERA } & \text { TRAY+FONDO } & \text { TRAY+META }\end{array}$

\section{Diferencias:}

Corte de la frase: cambio de ritmo y estilo.

La expresión à champ travers tiene un matiz diferente: manera (rapidez y por un atajo).

Es importante señalar la aportación de los estudios basados en datos empíricos, como los de Slobin $(1988,1991)$, que recogen producciones orales de locutores de varias lenguas, sobre todo, los estudios sobre adquisición de la lengua materna, aunque posteriormente su metodología ha sido aplicada al estudio de la adquisición de la lengua extranjera y al estudio de las traducciones (Cifuentes-Férez \& Rojo, 2008). Siendo producciones oral a partir de imágenes, estos datos evitan la influencia de otra lengua sobre la producción, de modo que se pueden identificar los esquemas de conceptualización dependientes de cada lengua. Como resultado, se pudo ampliar la clasificación binaria de Talmy y pasar a considerar un continuo de estructuras de conceptualización y lexicalización del movimiento, así como considerar factores pragmáticos (Slobin, 2004).

Para abordar pues adecuadamente algunos de los ejemplos que se nos presentan, en un enfoque contrastivo, de corte cognitivo, se hace necesario explicitar otro nivel, dado que, como hemos comprobado, estamos ya desbordando el nivel de frase/enunciado. Necesitamos 
por tanto introducir el nivel textual, es decir, en el nivel en el que se construye la coherencia y la cohesión a lo largo de diversos enunciados que construyen no solo secuencias textuales (Adam, 1997), sino discursos completos (unidades que corresponden a géneros).

Desde el momento en que utilizamos este tipo de datos de "uso" en contexto (corpus), el ejemplo tipo de Talmy ha de tomarse con cuidado, por cuanto, en el discurso real, $L a$ botella salió de la cueva flotando podría reducirse a La botella salió de la cueva o incluso a La botella salió puesto que, probablemente, el relato previo a la emisión de esta frase, ya nos ha indicado que la botella está en el mar o en una cueva ( $c f$. más adelante las funciones textuales del movimiento referencial, apartado 5). Así, en español o francés simplemente se mencionará el cambio de espacio, sin necesidad de mencionar, por "redundante" (porque se sobreentiende en el discurso) la manera del movimiento (float), o el origen del movimiento (out of the cave). En cambio, sería necesario explicitarlo en inglés, probablemente porque estas partículas son las encargadas de marcar la telicidad. De modo que el verbo español o francés (salir/sortir) solo trasladaría la información básica y nueva de que hay un desplazamiento hacia afuera (out).

Hablábamos anteriormente de "redundancia" y vemos ahora cómo este concepto cobra especial importancia por el hecho de que, en cada lengua, lo que se considera redundante y lo que se omite porque se "sobreentiende" es relativo. Así, una parte es relativa por el discurso anterior o posterior a un enunciado; otra, por nuestro conocimiento del mundo y otra puede estar lingüístico-cognitivamente codificada en la lengua.

\section{Combinatoria en el discurso: fenómenos en el eje sintagmático}

El fenómeno de la "combinatoria" suele ser comprendido como "combinatoria léxica" (Alonso, 1995, 2017), es decir, combinatoria de palabras con significado léxico. Sin embargo, cuando trabajamos en un enfoque contrastivo tomando en consideración diferentes lenguas, reducirnos a la combinatoria léxica puede mermar la utilidad del diccionario de producción/ traducción, ya que hay rasgos semánticos que, en una lengua, se expresan por medio de material léxico, mientras que, en otras, se utilizan medios morfosintácticos (ejemplo (4): el prefijo re- marca el valor de repetición de la acción, mientras en español el valor lo marca un verbo, volver) o incluso fonéticos (en el ejemplo (5), las expresiones quand même vs. pero...¿no?, además de tener categorías léxicas diferentes, marcan una entonación de frase diferente). La expresión de un rasgo semántico o un valor en una lengua pone en juego elementos muy diversos y transcategoriales. Aun tratándose de elementos léxicos, lo que en una lengua se expresa con un tipo de palabra, en otra puede variar, como muestran los ejemplos (4) y (5). 
(4)

4.a Il est rené.

4.b Ha vuelto a nacer.

(5)

5.a [en una réplica] Ben, il a quand même réussi son examen.

5.b Bueno, pero ha aprobado su examen, ¿no?

Recordaremos, además, que ni la frecuencia es necesariamente un factor para determinar una combinatoria restringida, ni tampoco la contigüidad inmediata de las unidades léxicas combinadas.

Veamos qué tipo de combinatoria queremos abordar en relación con los elementos que pueden entrar en juego en la expresión de un movimiento. No puede tratarse solo una colocación léxica de términos tomados dos a dos, como Tutin \& Grossmann, la describen:

\footnotetext{
Une collocation est l'association d'une lexie (mot simple ou phrasème) L et d'un constituant $\mathrm{C}$ (généralement une lexie, mais parfois un syntagme, par exemple à couper au couteau dans un brouillard à couper au couteau) entretenant une relation syntaxique telle que:

- C (le collocatif) est sélectionné en production pour exprimer un sens donné en cooccurrence avec L (la base);

- le sens de L est habituel. (Tutin \& Grossmann, 2002: 5)
}

Para los verbos, en general, interesa considerar también la combinatoria de elementos léxicos con elementos gramaticales o coligación:

Le concept de colligation, encore relativement original, me semble être à distinguer de la "simple" collocation grammaticale : elle porte sur les phénomènes de préférences régulières entre les unités lexicales ou grammaticales et 1) les fonctions ou catégories syntaxiques, 2) l'expression d'une catégorie sémantique grammaticalisée (modalité, aspect, négation...), 3) les positions dans la proposition, voire dans le texte. (Legallois, 2012: 50)

La coligación también forma parte de lo que querríamos poder trasladar al diccionario en lo relativo a los verbos de movimiento. Los verbos de movimiento, no solo por ser verbos, sino por su clase semántica, se prestan más al estudio de la estructura argumental de la frase, así como del orden de palabras o la combinatoria con preposiciones. Entre otras cosas, las especificidades tipológicas en la expresión del movimiento conciernen no solo a la combinación de verbos y preposiciones, sino también a la aparición (o no) de algunos sintagmas con información espacial en la frase (meta, origen, trayectoria) o de la información sobre la manera del movimiento, así como es también importante la cantidad de sintagmas con información espacial que pueden confluir en una sola proposición, es decir en una frase simple vs. en una frase compleja. 
Lo tipológicamente importante y específico en la combinatoria de estos verbos no es, por tanto, necesariamente, de tipo “colocación léxica”, como la del ejemplo (6),

(6) Corrió como un loco / Il a couru comme un fou.

sino que concierne aspectos como la "saturación” de slots o puestos semántico-sintácticos libres en la proposición, es decir, cuántos sintagmas diferentes pueden aparecer en un predicado en posición de información nueva afirmada (ejemplo (3)).

De modo que queremos poder describir también la coligación de una palabra que permita construir una frase aceptable con ella, es decir, que puede implicar más de dos elementos. Así, para nosotros, la dimensión del fenómeno combinatorio no solo es sintagmática, ni de tipo binario, sino proposicional, frástica o incluso discursivo -textual, como mostraremos más adelante.

En el caso que nos ocupa, la combinatoria en relación con los verbos de movimiento es compleja por varias razones:

1) desde el punto de vista de un diccionario combinatorio "de restricciones léxicas", la inclusión en el diccionario de verbos como correr, saltar, ir, venir // courir, sauter, aller, venir, en su significado más literal de movimiento, no parece en absoluto necesaria, por lo previsible de la combinatoria (de hecho, no los encontramos ni en REDES ni en LR, ni en DEC, ni en GDdesCOOC, en cambio sí en PRÁCTICO). Quien corre o salta o va o viene es una entidad dotada de movimiento (persona, animal) y, con nuestro conocimiento del mundo, esto no parece entrañar dificultad alguna para componer una frase. Los significados "literales" de los verbos de movimiento parecen a primera vista obvios y parecerían corresponder a reglas de subcategorización de los verbos y a nuestro conocimiento del mundo, más que a combinatoria restringida como la de las colocaciones;

2) como nos encontramos en la interfaz sintaxis-semántica y con la combinatoria de palabras en el eje sintagmático, empezamos a entrar en el terreno de la "gramática" (uso de preposiciones, por ejemplo) pero ligado al léxico;

3) el contraste "menor" en este ámbito entre lenguas cercanas (francés-español) frente, por ejemplo, al inglés, hace que podamos pensar que no se necesita registrar esta información en un diccionario bilingüe francés-español, como sí podría resultar necesaria en un diccionario bilingüe inglés-español o inglés-francés, donde la correspondencia entre unidades léxicas no se puede hacer fácilmente dado que la lexicalización de un evento de movimiento en inglés vs. lengua románica implica "redistribuir" el contenido conceptual o semántico en categorías léxicas o gramaticales diferentes. Pero... la simetría total entre dos lenguas no existe (ni siquiera siendo de la misma familia);

4) por último, habría que plantearse probablemente una distinción más afinada entre dos clases de verbos de movimiento: los verbos de manera de movimiento que tienen características temporales propias de las "actividades" (correr, saltar, nadar) y los verbos de despla- 
zamiento en muchos casos télicos (ir, venir, salir, entrar, bajar, subir, llegar, partir). En este sentido, algunos de los ejemplos para el francés que proponen Pourcel \& Kopecka (2005) o Kopecka (2006) servirían para tratar con más detalle los verbos de actividad con todos sus esquemas de conceptualización y lexicalización. No lo haremos aquí por falta de espacio.

\title{
4. Mise en texte de los verbos de movimiento o la referencia a un evento de movimiento en el texto: entre la lengua y el discurso
}

Slobin ya nos indicaba que los patrones de conceptualización/lexicalización interactúan con otros factores como la modalidad del discurso (escrito, oral, lengua de signos), la memoria o las prácticas culturales:

\begin{abstract}
A language provides its speakers with a range of ways of describing motion eventscombinations of lexical items and grammatical morphemes in various construction types. In attempting to define patterns of rhetorical style, it is also necessary to consider language-processing capacities (short-term memory, cognitive load), paralinguistic factors (voice quality, gesture), cultural practices, and modality (speech, writing, sign language). (Slobin, 2004: 220)
\end{abstract}

La propia lengua tiene intrínsecamente dos polos (Mondada \& Dubois, 1995), uno de creatividad o inestabilidad y uno de prototipicidad o estabilidad, entre los que oscila. Los propios locutores nativos podrán oscilar entre los dos polos dentro del margen que les permita su lengua materna con estilos estándar vs. propios o cambiantes. Por esto mismo es importante que el contraste entre las lenguas no se convierta en una "simetrización", aunque tratemos “en paralelo" dos lenguas (Sanz Espinar, 2004).

Así visto, podríamos preguntarnos hasta qué punto podemos volcar en el diccionario información "sistemática" pertinente sobre la dimensión textual y discursiva (la mise en texte o la mise en discours), de cara a la comprensión o expresión de eventos de movimiento en las distintas lenguas. Para ello, introduciremos dos grupos de nociones. Por un lado, las funciones textuales relativas al movimiento referencial que se produce de un enunciado a otro en el texto (cohesión), según lo presentan Klein \& Von Stutterheim (1991) y Von Stutterheim \& Klein (1989): introducción, mantenimiento, reintroducción y desplazamiento en un dominio referencial (espacialidad, temporalidad, entidades, eventos, modalidad) Por otro lado, las máximas del discurso (cantidad, calidad, pertinencia y modo) de Grice (1975), relativas a la coherencia. Ambas dimensiones se combinan para dar distintos tipos de resultado en un discurso real. Estas funciones deberían guiarnos a la hora de introducir ejemplos comparables (producidos independientemente en la L1 y la L2) y paralelos (L1 y traducción en L2) en el diccionario de tipo frástico y también superior al frástico: secuencias de frases donde se puedan percibir estos elementos.

Para las funciones textuales, nos basaremos en el modelo de la Quaestio de Klein \& Von 
Stutterheim 1991 y Von Stutterheim \& Klein (1989), según el cual un texto coherente es la respuesta a una quaestio, o pregunta abstracta como ¿Qué pasó después de que me fuese? ¿Qué hay en esta imagen? Estas quaestio permiten:

- $\quad$ evaluar el grado de coherencia del discurso creado (el discurso ha de responder a una intención principal de comunicación),

- distinguir un primer plano discursivo (que responde directamente a la quaestio), y un segundo plano que responde subsidiariamente a otras preguntas secundarias,

- identificar lo que los autores llaman el movimiento referencial, es decir, funciones textuales (FT) que entrañan diferentes modos de expresión: introducción de la referencia por primera vez en el discurso (FT-I), mantenimiento de la referencia (FT-M), reintroducción de una referencia anterior, con una discontinuidad (FT-R), o desplazamiento de la referencia (FT-D).

Por otro lado, las máximas de Grice, que se reagrupan bajo la etiqueta de principio de cooperación, orientan al locutor para adecuar su conversación a la situación comunicativa donde tenga lugar, a los requisitos que marque el propósito de la comunicación y a quién se dirige el locutor. De este modo, la cantidad, pertinencia y cualidad de la información (selección de la información) han de adecuarse, así como el modo de dirigirse al interlocutor respetando las reglas de cortesía.

Podemos ver algunos casos de cómo varía la expresión de la información según la función textual implicada en la referencia a un marco espacial en los extractos de producción oral de los ejemplos 7 a 9:

(7)

7.a El niño busca a su rana en un árbol. [FT-I]

Sube al árbol [FT-M], pero hay una colmena y las abejas salen enfadadas persiguiéndole

7.b L'enfant cherche sa grenouille dans un arbre. [FT-I]

Il $y$ [FT-M] grimpe, mais il y a une ruche et les abeilles fâchées le poursuivent fâchées

(8)

8.a ...y después se despiden y él se va.

Sale a la calle [FT-I] y cruza corriendo la avenida [FT-D]

Va $a$ un restaurante [FT-I] a comer

8.b ....après ils prennent congé et il s'en va

Il sort dans la rue [FT-R] et traverse l'avenue [FT-D] en courant

Il va manger dans un restaurant [FT-I] / Il va manger au restaurant / Il va dans un restaurant (pour manger) [explicitar aquí pour manger, sería un pleonasmo]

(9)

9.a En aquel bosque encuentran una casa. [FT-I]

Entran ø [FT-M] y descubren unos amuletos. 
9.b Dans cette fôret il trouvent une maison [FT-I] dans laquelle [FT-M] ils rentrent et ils $y$ [FT-M] découvrent des porte-bonheurs.

Dans cette fôret il trouvent une maison [FT-I]. Ils $y$ [FT-M] rentrent et ils (y/ø) [FT-M] découvrent des porte-bonheurs. [la duplicación de $y$ sería muy reiterativa]

\section{Tratamiento de la clase semántica de verbos de movimiento en TACTICOMB}

Sin tener aún el diseño concreto y electrónico del diccionario que proyectamos, sabemos que en todo diccionario hay que tomar decisiones en los niveles de: macroestructura (listado de los vocablos que va a integrar), la microestructura (ordenación de la información relativa a la lexía o bien a las distintas lexías correspondientes a un mismo vocablo) y, en nuestro caso, especialmente también, la mesoestructura. Dejamos aparte por ahora aspectos periféricos (ayuda del diccionario) y/o técnicos como el acceso a consultas-motor de búsqueda.

Una vez establecido que deseamos que los verbos de desplazamiento figuren en la macroestructura, en este artículo hemos hablado sobre todo de la microestructura y de la mesoestructura del diccionario. Algunos apartados de la mesoestructura han sido presentados ya y hemos tratado algunas especificidades de la clase semántica de los verbos de movimiento-desplazamiento. En la microestructura se crearían reenvíos a otros verbos de la clase semántica a la que pertenezca la unidad léxica tratada. Además, se cuidaría la homogeneidad en el tratamiento de la clase semántica global, que ha de ser uniforme tanto en el nivel semántico como en el sintáctico (Lépinette, 1996), tal y como indican también Mel'čuk \& Milićević:

A description of LUs done by semantic fields highlights their semantic similarities and helps achieve a degree of descriptive coherence much higher than would be possible if the choice of LUs to describe were arbitrary (according to alphabetical order, for instance). (...) In a dictionary, all the vocables within a given lexical field need to be described in a uniform way. (Mel'čuk \& Milićević, 2020: 200)

Así, la microestructura para los verbos del movimiento se organizaría como sigue ${ }^{10}$ :

ENTRADA

- Información gramatical del vocablo.

- Nivel de MCERL.

SYNSETS. Grupos de (cuasi-)sinónimos dentro de una clase semántica, separados para cada UL. Formaría parte de la mesoestructura.

10 En el DECFC, la macroestructura se refiere al artículo lexicográfico relativo al vocablo entero (para Mel'čuk et al. (1984-99) y Mel'čuk (2015) el vocablo es la entrada que reúne varias lexías). Para nosotros la macroestructura es la selección general de entradas, que se relaciona con la mesoestructura, dado que puede concernir entre otros la selección de las clases semánticas que vayan a incluirse y las unidades léxicas específicas para cada clase semántica; la microestructura que corresponde al artículo lexicográfico entero, aunque hay diferentes apartados dentro de éste, incluyendo el vínculo a la mesoestructura. 
- UL1. ES. La palabra de entrada seguida de sus cuasi-sinónimos bajo la etiqueta de una clase semántica (verbos de movimiento, verbos de movimiento-desplazamiento). Por ejemplo: verbos de movimiento-desplazamiento-IN <entrar, adentrarse, penetrar, introducirse $>$.

- UL1. FR. El equivalente de traducción más inmediato y sus cuasisinónimos bajo la etiqueta de la clase semántica equivalente en la L2. Por ejemplo: verbos de movimientodesplazamiento-IN <entrer, rentrer, pénétrer, s'introduire>.

DEFINICIÓN(ES) de las diferentes UL $\left(\mathrm{UL}_{1}, \mathrm{UL}_{2}, \mathrm{UL}_{3}\right)$ y remisión al apartado específico de cada UL.

CLAVES SEMÁNTICAS. Contraste entre una UL y sus cuasi-sinónimos o entre las diferentes UL del mismo vocablo. Formaría parte de la mesoestructura.

\section{ESQUEMAS DE CONCEPTUALIZACIÓN Y ESQUEMAS DE LEXICALIZA-} CIÓN propios de la clase semántica (verbos de movimiento, verbos de movimiento-desplazamiento). Eventualmente, complementación con grabaciones de microescenas de movimiento.

I. $\mathrm{N}_{0}+\mathrm{V}_{\text {MOV }}{ }^{11}$

I.a $\mathrm{N}_{0}+\mathrm{V}_{\text {MOV }}$ (MANERA O DESPLAZAMIENTO)

Jean court. ( $\mathrm{V}_{\text {MAN-MOV }}$ característica, il est coureur)

*Félix monte $\left(\mathrm{V}_{\mathrm{DEPL}}\right.$ no interpretable como característica)

\section{I.b $\mathrm{N}_{0}+\mathrm{V}_{\text {MOV }}$ (MANERA)}

On se précipita. (elisión circunstancial del $\mathrm{CIRC}_{\mathrm{ESP}}$, dependiendo del cotexto)

$$
\text { II. } \mathrm{N}_{0}+\mathrm{V}_{\mathrm{MOV}}+\left(\mathrm{CIRC}_{\mathrm{ESP} 1}\right) / \mathrm{N}_{0}+\mathrm{PR}_{\mathrm{CL} 1}\left(\mathrm{CIRC}_{\mathrm{ESP1}}\right)+\mathrm{V}_{\mathrm{MOV}}
$$

II. $\mathrm{N}_{0}+\mathrm{V}_{\mathrm{MOV}}+\left(\mathrm{CIRC}_{\mathrm{ESP1}}\right)\left(\mathrm{CIRC}_{\mathrm{ESP} 2}\right)\left(\mathrm{CIRC}_{\mathrm{ESP} 3}\right)$

Le chien est monté dans la voiture.

L'enfant a sauté d'un arbre à l'autre.

Il est passé par la fenêtre.

Le chat courait dans la chambre

II.b. $\mathrm{N}_{0}+\mathrm{PR}_{\mathrm{CL} 1}\left(\mathrm{CIRC}_{\mathrm{ESP} 1}\right)+\mathrm{V}_{\mathrm{MOV}}$

Le chien y est monté.

III. $\mathrm{N}_{0}+\mathrm{V}_{\mathrm{MOV}}+\mathrm{N}_{1}+\left(\mathrm{CIRC}_{\mathrm{ESP} 1}\right) / \mathrm{N}_{0}+\mathrm{PR}_{\mathrm{CL} 1}+\mathrm{V}_{\mathrm{MOV}}+\left(\mathrm{CIRC}_{\mathrm{ESP} 1}\right)$

$\overline{11} \mathrm{~N}_{0 \text { : }}$ primer sintagma nominal de la frase (sujeto), $\mathrm{N}_{1}$ : primer sintagma nominal del predicado; $\mathrm{V}_{\mathrm{Mov}}$ : verbo de movimiento (de manera- $\mathrm{V}_{\mathrm{MAN}-\mathrm{MOV}}$ o desplazamiento- $\mathrm{V}_{\mathrm{DEPL}}$ ); $\mathrm{PR}_{\mathrm{CL} 1}$ : primer pronombre clítico en la frase (en español y francés preceden al verbo conjugado en general); $\mathrm{CIRC}_{\mathrm{ESP} 1}, \mathrm{CIRC}_{\mathrm{ESP} 2}$ primer, segundo... complemento circunstancial de espacialidad de la frase; $\mathrm{CIRC}_{\mathrm{MAN}}$ : complemento circunstancial de manera. 
III.a $\mathrm{N}_{0}+\mathrm{V}_{\mathrm{MOV}}+\mathrm{N}_{1}+\left(\mathrm{CIRC}_{\mathrm{ESP} 1}\right)\left(\mathrm{CIRC}_{\mathrm{ESP} 2}\right)$

Il a traversé le pays du nord au sud.

III.b. $\mathrm{N}_{0}+\mathrm{PR}_{\mathrm{CL} 1}+\mathrm{V}_{\mathrm{MOV}}+\left(\mathrm{CIRC}_{\mathrm{ESP} 1}\right)\left(\mathrm{CIRC}_{\mathrm{ESP} 2}\right)$

Il l'a traversé du nord au sud.

IV. $\mathrm{N}_{0}+\mathrm{V}_{\mathrm{MOV}}+\left(\mathrm{CIRC}_{\mathrm{ESP} 1}\right)\left(\mathrm{CIRC}_{\mathrm{MAN}}\right) / \mathrm{N}_{0}+\mathrm{PR}_{\mathrm{CL} 1}+\mathrm{V}_{\mathrm{MOV}}+\left(\mathrm{CIRC}_{\mathrm{MAN}}\right)$

IV.a. $\mathrm{N}_{0}+\mathrm{V}_{\mathrm{MOV}}+\left(\mathrm{CIRC}_{\mathrm{ESP1}}\right)\left(\mathrm{CIRC}_{\mathrm{MAN}}\right)$

Il est sorti du cinéma en pleurant.

Il a traversé la rue en courant.

IV.b. $\mathrm{N}_{0}+\mathrm{PR}_{\mathrm{CL} 1}+\mathrm{V}_{\mathrm{MOV}}+\left(\mathrm{CIRC}_{\mathrm{MAN}}\right)$

Il en est revenu en pleurant.

Il y est monté en courant.

IV.c $\mathrm{N}_{0}+\mathrm{V}_{\mathrm{MOV}}+\mathrm{N}_{1}+\left(\right.$ CIRC $\left._{\mathrm{MAN}}\right)$

Mélanie monte les escaliers en courant.

V. $\mathrm{N}_{0}+\mathrm{V}_{\mathrm{MOV}}+\mathrm{N}_{1}+\left(\mathrm{CIRC}_{\mathrm{ESP1}}\right)\left(\mathrm{CIRC}_{\mathrm{ESP} 2}\right)\left(\mathrm{CIRC}_{\mathrm{MAN}}\right)$

Il a mis les clés dans sa poche sans faire du bruit.

ESQUEMAS DE CONCEPTUALIZACIÓN y LEXICALIZACIÓN ESPECÍFICOS DE LA UNIDAD LÉXICA O LEXÍA retomando la clasificación precedente (I-V).

Se trataría también de una parte de la mesoestructura que se realizaría mediante la comparativa de frases analizadas en cada lengua en términos de esquema de conceptualización tal como lo presentamos en el ejemplo (3) de nuestro artículo.

\section{EJEMPLOS GENERALES RELATIVOS A LA UTILIZACIÓN EN DISCURSO DE LAS UNIDADES.}

Secuencias textuales semejantes a los ejemplos (7-9) presentados anteriormente.

\section{Conclusiones}

Tras este breve estudio del tipo de combinatoria que podemos presentar de la clase semántica de los verbos de movimiento en el diccionario para una adecuada comprensión, aprendizaje, producción y traducción de los mismos, hemos visto que esa combinatoria sería, en primer lugar, más propia de lo que habitualmente se entiende por coligación o incluso reglas de subcategorización de los verbos. 
El reto es significativo y podríamos sintetizarlo en la siguiente pregunta: ¿En qué medida sistematizamos en un diccionario una especie de "gramática para la producción de un texto en una L1 vs. una L2" a través de las unidades léxicas (verbos) implicadas en la expresión de un evento de movimiento? Para construir este léxico-gramática cognitivo hemos encontrado varios problemas:

a) el problema de la articulación del eje paradigmático (clase semántica de verbos de movimiento) con el eje sintagmático (construcción de un enunciado válido),

b) el problema de una descripción "cómoda" de la interfaz sintaxis-semántica (cómo presentar la información de modo relativamente accesible o intuitivo para el gran público),

c) cómo gestionar la interfaz entre lengua y discurso: cómo construir dicho enunciado válido en distintos contextos con equivalencia semántico-pragmática (teniendo en cuenta variables diferentes, dando soluciones posibles para distintos casos, según principios textuales y discursivos de cohesión y coherencia),

d) y, por último, la interfaz L1 - L2, es decir, cómo poner en paralelo las opciones equivalentes "exactas" y/o más cercanas (entendiendo por "cercanas" no necesariamente calcos, sino adecuadas pragmáticamente, por tanto, no necesariamente lexicalizadas), que habitualmente en los diccionarios se simplifican o se omiten (dicha opción resultaría para nosotros demasiado reductora).

La expresión del movimiento (ligada a la expresión de la espacialidad y a la manera) está determinada también a nivel textual, de modo que, al construir un discurso, podemos preguntarnos si conceptualizamos la información en una o más proposiciones $\mathrm{y}$, por tanto, si la formulamos en una frase simple o dos o en una principal acompañada de subordinada o en dos simples. Por ello, hemos previsto la inclusión de pequeñas secuencias textuales en el diccionario, tanto paralelas (originales y sus traducciones) como comparables (originales comparables creados independientemente en cada una de las dos lenguas). Así, dada la importante repercusión de la dimensión textual en la expresión del movimiento, hemos considerado la presencia de ejemplos más extensos que una sola frase o enunciado simple o compuesto. Y, en la selección de estos ejemplos de dimensión suprafrástica, hemos propuesto marcar las funciones textuales o discursivas que determinan ciertas opciones a la hora de explicitar o no la información, determinadas por la cohesión (movimiento referencial) y la coherencia (máximas del discurso).

Por último, cabe insistir en la ventaja, o incluso necesidad, del estudio de las clases semánticas en bloque para poner de relieve combinatoria sistemática dentro de dicho grupo de palabras.

Esto probablemente derivará en que TACTICOMB describa combinatoria de distinto tipo: a veces, más de tipo léxico, a veces más de tipo coligación o mixta, según las caracterís- 
ticas de las clases semánticas y de los contrastes interlingüísticos, circunstancia que iremos analizando conforme la vayamos encontrando para delimitar lo que sería el núcleo común a todo el diccionario.

En cualquier caso, creemos haber planteado las líneas maestras del diccionario con suficiente claridad. La organización de la microestructura (esto ya ocurre en otros diccionarios combinatorios) puede ser diferente según el tipo de entrada y, por otro lado, hemos introducido el nivel de la mesoestructura, que podrá tomar la forma de cuadros sintéticos de clases semánticas de palabras o incluso ser complementado, en el caso de los verbos de movimiento, con pequeñas escenas de vídeo.

Tal como lo visualizan Bylund \& Athanopoulos (2015: 9)

\begin{abstract}
in order to facilitate the internalisation of a novel concept, teaching should not only include linguistic descriptions of the concept, but also engage students in activities that allow them to process the concept in modalities that do not relate to language. Such activities may include exercises of grouping or sorting pictures depicting colours, objects, or motion according to different parameters (see also Jarvis \& Pavlenko, 2008). Multimodal teaching strategies (e.g., visualisation of aspectual relations) are to some extent already integrated in language teaching (e.g., Lantolf, 2010; Pavlenko \& Driagina, 2006; van Compernolle, 2011, 2013; Williams et al., 2013). The growing body of evidence on multimodal conceptual representation not only strengthens the theoretical rationale for existing practices, but also constitutes a starting point for future endeavours targeted at developing language teaching methods.
\end{abstract}

Dado que se ha señalado que los vídeos cortos pueden ser elementos para el aprendizaje de las características de estas lexicalizaciones, podemos reflexionar sobre si sería un complemento adecuado para la comprensión, el aprendizaje, la traducción y la producción para el caso de la clase de verbos de movimiento en el diccionario bilingüe. Podría parecer que la comprensión es menos importante que otras utilizaciones del diccionario, pero creemos que la comprensión ha de preceder todas las demás operaciones y, mediante el vídeo, se permitiría captar cómo se ha "seleccionado" la información para expresar un evento de movimiento "real" en una lengua sin tener que pasar por otra lengua. En la época actual en que los medios electrónicos son más accesibles, creemos factible plantearnos, sin pecar de excesiva ambición, este complemento al diccionario. Con la imagen en movimiento, tendríamos un punto de fuga que nos permitiría no depender enteramente de una lengua para acceder a la conceptualización de otra, ya que al traducir o pasar de una lengua a otra "sin la experiencia real" de la comunicación en contexto, arrastramos o calcamos algunos matices de significado de la L1 que nos influye a la hora de comprender o utilizar la L2.

Como colofón, no podemos dejar de mencionar que un diccionario como el que proponemos es un nuevo concepto de diccionario. Como todo diccionario combinatorio, es una entidad todavía bastante desconocida para el gran público. Dar a conocer, aprender a usar estos diccionarios que funcionan como léxicos-gramática cognitivos e idear tareas muy con- 
cretas de comprensión, aprendizaje, traducción o producción con ellos es otra historia que aún ha de ser contada, de modo que a la vez que tratamos de allanar el camino hacia conceptos lingüístico-cognitivos que reciben a veces denominaciones complejas, asumimos la necesidad de una formación del profesorado para la comprensión del fenómeno, la selección de materiales y la elaboración de actividades innovadoras. Esperamos que nuestra propuesta de organización de la información lingüística y cognitiva en un nuevo formato lexicográfico ayude en esta tarea.

\section{Referencias bibliográficas}

ADAM, Jean-Michel. 1997. "Genres, textes, discours: pour une reconception linguistique du concept de genre" in Revue belge de philologie et d'histoire $\mathrm{n}^{\circ} 75(3), 665-681$. <http://www. persee.fr/doc/rbph_0035-0818_1997_num_75_3_4188> [21/05/2020].

Alonso, Margarita. 1995. "Hacia una definición del concepto de colocación: de J. R. Firth a I. A. Mel'čuk" in Revista de Lexicografia no 1, 9-28. <https://doi.org/10.17979/ rlex.1995.1.0.5693> [12/05/2020].

Alonso, Margarita. 2001. "Construction d'une base de données des collocations bilingue français-espagnol” in Langages $\mathrm{n}^{\circ} 143,5-27$. <https://doi.org/10.3406/lgge.2001.888https:// www.persee.fr/doc/lgge_0458726x_2001_num_35_143_888> [15/05/2020].

Alonso, Margarita. 2017. "Diccionarios combinatorios" in Estudios de Lingüística del Español no 38, 173-201 <https://www.raco.cat/index.php/Elies/article/view/327486> [21/05/2020].

BARrios, María. 2008. "Diccionarios combinatorios del español: diferencias y semejanzas entre Redes y Práctico" in Azorín, Dolores (dir.). El diccionario como puente entre las lenguas y culturas del mundo. Actas del II Congreso Internacional de Lexicografía Hispánica, Alicante, Biblioteca Virtual Miguel Cervantes, 197-203. <http://www.cervantesvirtual.com/ nd/ark:/59851/bmcn5922> [11/05/2020].

Berman, Ruth \& Dan Slobin. 1994. Different ways of relating events in narrative: a crosslinguistic developmental study, vol. 1. Hillsdale, Lawrence Erlbaum.

Bylund, Emanuel \& Panos Athanasopoulos. 2015. "Introduction: Cognition, Motion events and SLA" in The Modern Language Journal n ${ }^{\circ}$ 99, 1-13. <DOI: 10.1111/modl.12175>.

Cifuentes Férez, Paula \& Ana Rojo. 2008. “Thinking for translating: A think-aloud protocol on the translation of manner of motion verbs. Target $\mathrm{n}^{\circ} 27(2), 273-300$. <DOI: 10.1075/ target.27.2.05cif $>$.

Grice, Paul. 1975. "Logic and Conversation" in Grice, Paul. 1989. Studies in the Way of Words. Harvard, Harvard University Press, 22-40.

Hickmann, Maya, Henriette Hendriks \& Françoise Roland. 1998. "Référence spatiale dans les récits d'enfants français: perspective inter-langues" in Langue Française $\mathrm{n}^{\mathrm{o}} 118$, 104-124. 
Klein, Wolfgang \& Christiane Von Stutterheim. 1991. "Text structure and referential movement" in Sprache und Pragmatik n 22, 1-31.

KopecKA, Annette. 2006. "The semantic structure of motion verbs in French: Typological perspectives" in Hickmann, Maya \& Stéphane RoberT. (eds.). Space in languages: Linguistic Systems and Cognitive Categories. Amstedam, John Benjamins.

Legallois, Dominique. 2012. "La colligation: autre nom de la collocation grammaticale ou autre logique de la relation mutuelle entre syntaxe et sémantique?" in Corpus n ${ }^{\circ} 11,31-54$. $<$ http://journals.openedition.org/corpus/2202> [11/05/2020].

LÉPINETTE, Brigitte. 1989. "Vers un dictionnaire explicatif et combinatoire bilingue. Bases théoriques et élaboration de quelques articles" in Cahiers de lexicologie n॰54, 105162. $<10.15122 /$ isbn.978-2-8124-4307-7.p.0105> [11/05/2020].

LePinette, Brigitte. 1996. "Le rôle de la syntaxe dans la lexicographie bilingue" in Bejoint, Henri \& Philippe Thoiron. Les dictionnaires bilingues. Paris, Duculot, 53-70.

LeVelt, Wilhem. 1989. Speaking: from Intention to Articulation, Cambridge Massachusetts, London, M.I.T. Press.

MeL'ČUK, Igor. 2015. Semantics. From meaning to text, vol. 3. Amsterdam, John Benjamins.

MeL'ČUK, Igor \& Jasmina MiLićEvić. 2020. An advanced introduction to semantics. A meaning-text approach. Cambridge, Cambridge University Press. Edición Kindle.

Mondada, Lorenza \& Danièle Dubois. 1995. "Construction des objets de discours et catégorisation : une approche des processus de référenciation" in TRANEL n 23, 273-302.

Morimoto, Yuko. 2001. Verbos de movimiento. Madrid, Visor.

Noyau, Colette et al. 2005. "Two dimensions of the representation of complex events structures: granularity and condensation. Towards a typology of textual production in L1 and L2" in HeNDRIKs, Henriette (ed.). The structure of learner language. Berlín, De Gruyter, $157-$ 201.

Pourcel, Stéphanie \& Anetta Kopecka. 2005. "Motion expression in French: typological diversity" in Durham \& Newcastle working papers in linguistics, $\mathrm{n}^{\circ} 11,139-153$.

RAE. 2009. Nueva gramática de la lengua española. Morfología y sintaxis. Madrid, Espasa.

SANZ EsPINAR, Gema. 2004. "El libro bifronte. la representación del francés y del español en el diccionario bilingüe” in SuÁrez, María Pilar et al. (eds.). L'autre et soi-même: La identidad y la alteridad en el ámbito francés y francófono. Madrid, UAM/Ima Ibérica, 445-454.

Sanz Espinar, Gema, Jin-NAm Choi \& HA-Sung Park. 2012. "Influence de l'input oral dans l'acquisition du FLE selon la distance typologique des Langues (espagnol $>$ français, coréen>français)" in Verba volant $\mathrm{n}^{\circ}$ 2(2), 43-64. <http://letras.ufpel.edu.br/verbavolant> [15/05/2020].

SANZ EsPinar, Gema \& Aránzazu GIL. 2019. "Vers un dictionnaire combinatoire bilingue français-espagnol: approche des mots renvoyant à la 'peur' en français et en espagnol à travers leur combinatoire lexicale et leur comportement dans de grands web-corpus" ponencia en XXVIII Congreso AFUE, 12 abril 2019, Universidad Autónoma de Madrid. 
SLobin, D. 2004. "The many ways to search for a frog: Linguistic typology and the expression of motion events" in STRÖMQVIST, Sven \& Ludo VERHOEVEN (eds.). Relating events in narrative. Vol. 2. Typological and contextual perspectives, Mahwah, NJ, Lawrence Erlbaum Associates, 219-257.

TALmy, Leonard. 1985. "Lexicalization patterns: semantic structures in lexical forms" in Shopen, Timothy (ed.). Language typology and syntactic description, vol. 3, Cambridge, University Press, 57-147.

Tutin, Agnès \& Francis Grossmann. 2002. "Collocations régulières et irrégulières: esquisse de typologie du phénomène collocatif" in Revue française de Linguistique appliquée, Lexique: recherches actuelles $\mathrm{n}^{\circ}$ VII (1), 7-25.

Von Stutterheim, Christiane \& Wolfgang Klein. 1989. "Referential movement in descriptive and narrative discourse" in Dietrich, RaIner \& Carl Graumann. (eds.). Language Processing in Social Context, Amsterdam, Elsevier, 39-76.

\section{Diccionarios ordenados por la abreviatura utilizada en el artículo}

[DECFC] MeL' ̌́uK, Igor et al. 1984-1999. Dictionnaire explicatif et combinatoire du français contemporain: Recherches lexico-sémantiques, Vol. I-IV. Montreal, Presses de 1'Université de Montréal.

[DICE] Alonso, Margarita. 2004. Diccionario de colocaciones del español. <http://www. dicesp.com/paginas $>[21 / 05 / 2020]$.

[EDCEI] Greco, Simone. 2015. Esbozo de un diccionario combinatorio español-italiano. Tesis doctoral. Universidad Autónoma de Madrid. Dirigida por Javier García González. $<$ https://repositorio.uam.es/handle/10486/672225> [21/05/2020].

[GDdesCOOC] Beauchesne, Jacques, Beauchesne, Maude \& Beauchesne, Kim. 2009. Le Grand Dictionnaire des cooccurrences. Québec, Guérin.

[PRÁCTICO] BosQue, Ignacio (dir.). 2006. Diccionario combinatorio práctico del español contemporáneo. Madrid, SM.

[REDES] BosQue, Ignacio (dir.). 2004. Redes. Diccionario combinatorio del español contemporáneo. Madrid, S.M. 